\title{
Simulation of the charge migration in DNA under irradiation with heavy ions
}

\author{
Oleg V. Belov ${ }^{\mathrm{a}}$, Denis L. Boyda ${ }^{\mathrm{b}}$, Ianik Plante ${ }^{\mathrm{c}}$ and Sergey Eh. Shirmovsky ${ }^{\mathrm{b} *}$ \\ ${ }^{a}$ Laboratory of Radiation Biology, Joint Institute for Nuclear Research, 6 Joliot-Curie Street, 141980 \\ Dubna, Moscow Region, Russia \\ ${ }^{b}$ Far Eastern Federal University, 8 Sukhanov Street, 690950 Vladivostok, Russia \\ ${ }^{c}$ Wyle Science, Technology and Engineering, 1300 Hercules Ave, Houston, Texas 77058, USA
}

\begin{abstract}
A computer model to simulate the processes of charge injection and migration through DNA after irradiation by a heavy charged particle was developed. The most probable sites of charge injection were obtained by merging spatial models of short DNA sequence and a single $1 \mathrm{GeV} / \mathrm{u}$ iron particle track simulated by the code RITRACKS (Relativistic Ion Tracks). Charge migration was simulated by using a quantum-classical nonlinear model of the DNA-charge system. It was found that charge migration depends on the environmental conditions. The oxidative damage in DNA occurring during hole migration was simulated concurrently, which allowed the determination of probable locations of radiation-induced DNA lesions.
\end{abstract}

Keywords: DNA damage, charge migration, radiation

\section{Introduction}

The phenomenon of charge transfer through DNA is a hot topic of many experimental and theoretical papers [1-5]. Some of these studies are concerned with the issues of the charge migration induced by environmental factors, among which ionizing radiation is of great interest $[6,7]$. Recently extensive research was performed on the identification of biophysical mechanisms leading to the formation of radiation-induced DNA lesions of different types [8-10]. In this regard, it is important to reveal the processes involved in charge transfer through the DNA molecule exposed to ionizing radiation for future investigations.

In the paper, the emphasis is placed on DNA damage resulting from ionizing radiation, more specifically by the high charge and energy (HZE) nuclei which are also called heavy ions. HZE usually refers to nuclei of atomic number $(Z)$ greater than 2 (helium). One important feature of heavy ions is their high-linear energy transfer (LET), which makes them more damaging for biological structures than sparsely ionizing radiations like $\gamma$ - and X-rays. The DNA damage caused by HZE nuclei frequently results in clustered DNA lesions of higher complexity which lead to more pronounced biological effects with regards to cell killing, induction of chromosome aberrations, structural gene muta-

\footnotetext{
* Address for correspondence: Sergey Eh. Shirmovsky, Far Eastern Federal University, 8 Sukhanov Street, 690950, Vladivostok, Russia. Tel: +7 914 7906601; Fax: +7 423 2432315; E-mail: shirmovskiy.sye@dvfu.ru.
} 


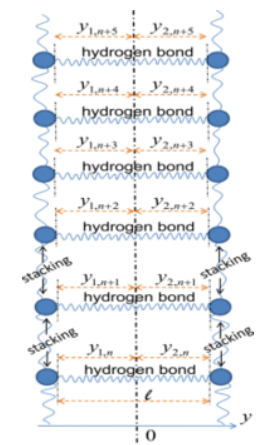

Fig. 1. Coupled harmonic oscillators representing the bases in a double-stranded model of DNA.

tions, initiation of cell transformation, and so on than exposure to low-LET ionizing radiations. Consequently, the charge migration is an important mechanism that follows DNA damage since it may influence the final lesion type and its complexity.

In this study, a numerical model of charge migration along DNA following both the direct and indirect actions of HZE particles was proposed. In the model, DNA is considered as a double-stranded molecule and represented as shown in Figure 1.

The model takes into account the stacking interaction in each DNA strand, the existence of hydrogen bonds between bases, and individual base positions. Since direct and indirect effects result in the loss of an electron within DNA, or the creation of a positive charge, we will speak about a hole as a carrier of a charge. The authors' model of this process yields a system of nonlinear differential equations for which the solution gives the time evolution of the probabilities of the charge locations and the oxidative damage of the DNA [11].

In the paper, the possible involvement of the sugar-phosphate backbone into the charge migration is not taken into consideration. At the moment, the influence of the sugar-phosphate backbone on the charge transfer is being discussed in several experimental and theoretical papers [12, 13]. These studies are based on the idea proposed by L. Brillouin, who noted that the sugar-phosphate backbone may have conductivity properties. However, according to the current consensus, much attention is devoted to the study of the charge migration through the DNA $\pi$-base stacks as proposed by D. Eley and D. Spivey [14].

\section{Method}

This model consists of three main parts. In the first part, all interactions of the HZE particle are simulated with water (the so-called radiation track structure). In the second part, the initial locations of the positive charges in a DNA sequence are obtained by finding the intersection points between the atoms of the sequence and both the direct ionizations and the radiolytic species of the track structure. In the third part, a quantum-classical model is applied to follow the charge migration in DNA.

\subsection{Track structure simulation}

To simulate the heavy ions' radiation track structure a Monte Carlo simulation code RITRACKS [15-17] was used. It provides stochastic track calculations of the energy deposition events, ionization and excitation of medium molecules, position and direction of the secondary electrons, and the coordi- 


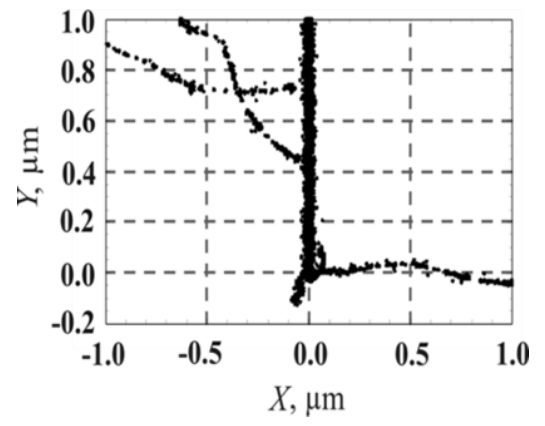

Fig. 2. XY projection of a $1 \mathrm{GeV} / \mathrm{u}{ }^{56} \mathrm{Fe}$ - ion track in liquid water as calculated by RITRACKS.

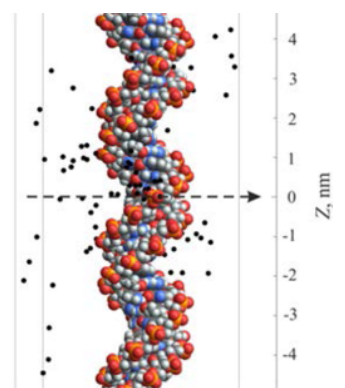

Fig. 3. The space-filling model of B-DNA combined with the $1 \mathrm{GeV} / \mathrm{u}{ }^{56} \mathrm{Fe}$ - ion track. The dots represent ionizations or radiolytic species produced in the medium. The dashed line shows the track axis and its direction.

nates of all radiolytic species. This code utilizes liquid water as a medium for the track structure simulation. To demonstrate the approach, a $1-\mathrm{GeV} / \mathrm{u}{ }^{56} \mathrm{Fe}$ ion $(\mathrm{LET} \sim 150 \mathrm{keV} / \mu \mathrm{m})$ was used. The track length is set to $1 \mu \mathrm{m}$ for this simulation (Figure 2). In the cases studied for this work, the ion trajectory's axe intersects the DNA strand perpendicularly as it is shown in Figure 3.

\subsection{Determination of charge injection sites}

To find the initial locations of the positive charges in the DNA strand, a computer program was written to determine the intersection points of the ionizations in the radiation track structure with a linear 68- bp B-DNA sequence (Figure 3), using the atomic radii values given in Table 1. The spacefilling model of DNA is obtained using the 3DNA software package [18].

The program written compares sequentially the positions of each direct ionization or radiolytic species with the coordinates of atoms approximated by spheres of the corresponding radii and identifies all atoms which are possibly affected by the radiation track. In this study, for simplification, the influence of the molecular dynamics of DNA on initial damage is not taken into consideration.

It is possible for many atoms of a given base, sugar or phosphate, to be ionized. However, DNA bases are on the whole considered as units of initial charge injection for the charge transfer model in question. When several of the sugar and/or phosphate atoms are ionized, it is assumed that these events are either a possible substrate for other types of DNA lesions or a limited number of incidents when the charge migration starts from the sugar-phosphate backbone and, therefore, cannot be described with this model. When more than one atom of a base is initially ionized, it is assumed that it is a single ionization of this base as a first approximation.

Table 1

The radii values of atoms of the DNA molecule

\begin{tabular}{|l|l|}
\hline Atom & United radius, $10^{-12}(\mathrm{~m})$ \\
\hline $\mathrm{H}$ & 120 \\
\hline $\mathrm{C}$ & 170 \\
\hline $\mathrm{N}$ & 160 \\
\hline $\mathrm{O}$ & 155 \\
\hline $\mathrm{P}$ & 195 \\
\hline
\end{tabular}




\subsection{Charge migration in a double-stranded DNA fragment}

The DNA-hole interaction is investigated using a mixed quantum-classical approach [4]. In this model, the total DNA-charge Hamiltonian $H$ is written as a sum of classical and quantum terms:

$$
H=H_{C l}+<\psi\left|H_{Q}\right| \psi>
$$

$H_{C l}$ being the classical part of the Hamiltonian:

$$
H_{C l}=\sum_{n=1}^{N}\left(\frac{M \dot{y}_{1, n}^{2}}{2}+\frac{M \dot{y}_{2, n}^{2}}{2}+\frac{k_{v}\left(y_{1, n}-y_{1, n-1}\right)^{2}}{2}+\frac{k_{v}\left(y_{2, n}-y_{2, n-1}\right)^{2}}{2}+\frac{k_{h}\left(y_{1, n}-y_{2, n}+l\right)^{2}}{2}\right) .
$$

In Eq. (2), the first and second terms correspond to the kinetic energy of the bases located on two different DNA strands. The third and fourth terms are the contributions of the potential energy from stacking interactions through each DNA strand while, the last term represents the potential energy of the hydrogen bonds between the bases. Furthermore, $M$ is the effective base mass, $k_{h}$ and $k_{v}$ are force constants, $l$ is the horizontal distance between the bases at equilibrium, and $N$ in the summation is the number of base pairs in the strand. In fact, the Hamiltonian $H_{C l}$ represents the DNA macromolecule as a system of coupled harmonic oscillators formed by two adjacent strands, which are joined to compose DNA (Figure 1). The second part of Eq. (1) is determined by the quantum dynamics of charged particles.

Two sets of parameters are introduced. The first is the ionization potentials, or bases' energies $\varepsilon_{n}$ which yields the energy profile of the DNA sequence. Guanine (G) possesses the smallest ionization potential, being approximately $0.4-0.45 \mathrm{eV}$ less than that of adenine (A) and $0.7-1.5 \mathrm{eV}$ less than those of thymine (T) and cytosine (C), respectively $[19,20]$. The second set of parameters is the coupling matrix elements $v_{n, m}$ which determine the rate of charge transfer between the bases $n$ and $m$. Since the bases' energies and coupling matrix elements are influenced by the structure and dynamics of DNA and by the DNA interaction with the environment (solvent), they are not static values.

The hole Hamiltonian $H_{Q}$ is determined by the tight-binding model. In this approach [4, 21, 22], $H_{Q}$ can be written as:

$$
H_{Q}=\sum_{n=1}^{N}\left(\alpha_{n}^{0}+\alpha_{n}^{\prime}\left(y_{1, n}-y_{2, n}+l\right)\right)|n><n|+\sum_{n \neq m}^{N} v_{\mathrm{n}, \mathrm{m}}|n><m|
$$

where $\mid n>(<m /)$ are the charged particle states in Dirac's notation. In the model, the matrix elements $v_{n, m}$ are static (i.e. time - independent). In general, the values of $v_{n, m}$ are different for the different hole transitions. As has been shown by many calculations (for a more comprehensive data set, see [23]), the matrix elements could differ, depending on the nucleobase types and the relative nucleobase positions on the DNA strand. Also, it was shown in previous works $[4,21]$ that this approach is a good approximation for the study of the nonlinear hole dynamics on the basis of the experimental results $[1,2]$. However, the bases' energies vary with time and are determined by the dynamics of DNA and by the DNA-environment interaction. In the model, the bases' energies are written as

$$
\varepsilon_{n}=\alpha_{n}^{0}+\alpha_{n}^{\prime}\left(y_{1, n}-y_{2, n}+l\right)
$$


in which the first term $\alpha_{n}^{0}$ is static and corresponds to the energy of an individual base while the second term changes with time. Thus, the interaction between the charged particle and DNA is introduced by the second component, $\alpha_{n}^{\prime}$ being a fitting parameter. The value of this parameter was obtained by the method of successive iterations, i.e. the values of $\alpha_{n}^{\prime}$ parameter were adjusted until the probability of the charge location on the initial base became less than 0.1 . The charge migration through DNA's base sequence is determined by the Schrödinger equation

$$
i \hbar \frac{\partial \mid \psi>}{\partial t}=H_{Q} \mid \psi>
$$

The solution to the Schrödinger Eq. (5) is expressed as

$$
\left|\psi>=\sum_{n=1}^{N} b_{n}(t)\right| n>.
$$

In Eq. (6), the squared module $P_{n}=\left|b_{n}(t)\right|^{2}$ represents the probability of the charge being located at the $n^{\text {th }}$ base at time $t$. The system of equations defining the DNA - charge dynamics is determined by the Schrödinger Eq. (5) and Hamilton equations for the bases located on either strand:

$$
\begin{aligned}
& M \frac{\partial^{2} y_{1, n}}{\partial t^{2}}=-\frac{\partial H}{\partial y_{1, n}}-\gamma \frac{\partial y_{1, n}}{\partial t} \\
& M \frac{\partial^{2} y_{2, n}}{\partial t^{2}}=-\frac{\partial H}{\partial y_{2, n}}-\gamma \frac{\partial y_{2, n}}{\partial t}
\end{aligned}
$$

$H$ being the total DNA-charge Hamiltonian Eq. (1). The interaction of DNA with the environment leads to energy dissipation, which is taken into account by inserting damping terms empirically (the last terms in Hamilton equations). In the model the damping coefficient $\gamma$ is linked to the viscosity of the medium $(\eta)$ by the Stokes' law $\gamma=6 \pi \eta r$, where $r=5 \AA$.

\section{Results and discussion}

\subsection{Evaluation of charge migration processes}

The two-dimensional projection of the radiolytic species created by a $1 \mathrm{GeV} / \mathrm{u}{ }^{56} \mathrm{Fe}$ track as simulated by RITRACKS is shown in Figure 2. For the calculations, one sample of the ${ }^{56} \mathrm{Fe}$ track and spacefilling DNA model were randomly selected (Figure 3). In this study, it is assumed that the spatial positions of atoms composing the DNA molecule and therefore the positions of the nucleobases are fixed in relation to the time. It means that in these calculations the time-independent DNA structure is used. Figure 4 schematically shows the initial locations of the charges created by the ionizing radiation.

The objective of this work is to identify possible regularities of charge migration along short DNA sequence and estimate a probable distribution of oxidative damage in DNA induced as a consequence of the presence of a charge and its transfer. The study assumes that the probability of charge location at a certain base correlates with the probability of the corresponding DNA cleavage. In other words, it is assumed that the final location of the charge may determine the most probable sites of DNA damage. This assumption is made on the basis of experimental studies [1,2], where the identification of specific DNA damage was correlated with the hole presence at certain DNA bases. 


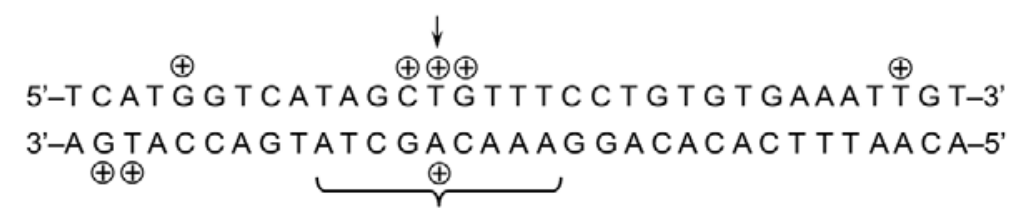

Fig. 4. The track-DNA interaction. The symbols $\oplus$ indicate the calculated sites of a charge injection. The arrow shows the position of the particle's track axis. The horizontal brace shows the DNA sequence selected for the charge migration analysis.

For the analysis of the charge migration, the A1 T2 C3 G4 A5 (+) C6 A7 A8 A9 DNA sequence was selected (Figure 4). In this notation, the letters are the base types, the numbers are their ordinal positions in the DNA sequence and the symbol $(+)$ indicates the hole position at the time of formation $(t=0)$. In this study, a charge transfer is simulated when a single charge migrates through the DNA sequence while other ones are assumed to be fixed. The distinctions between the intra-strand and interstrand charge transfer are not considered since it requires further development of the model.

For the simulation, the following parameters were used: $\alpha_{G}^{0}=1.24 \mathrm{eV}, \alpha_{A}^{0}=1.69 \mathrm{eV}, \alpha_{T(C)}^{0}=1.9$ $\mathrm{eV}$ [19]; $k_{v}=0.025 \mathrm{eV} / \AA^{2}, k_{h}=4.90 \cdot 10^{-3} \mathrm{eV} / \AA^{2}$ and $M=2.51 \cdot 10^{-25} \mathrm{~kg}$ [24]. The matrix element $v_{n, m}=$ $0.12 \mathrm{eV}$. This value is located in the interval of the possible matrix elements $v_{n, m}$ values as indicated in previous works [23]. At equilibrium, the average distance $l=7 \AA$.

As has been mentioned before, since the hole migration through DNA chain is strongly dependent on both the DNA dynamics and environment properties, an attempt was made to study the effect of the media by using two different values of the viscosity $\eta: 6.95 \cdot 10^{-4} \mathrm{~Pa} \cdot \mathrm{s}$ for an aqueous medium and $1.20 \bullet 10^{-5} \mathrm{~Pa} \bullet \mathrm{s}$ for the gas phase. Indeed, the calculations for the gas phase are important to study the effect of the parameters such as the viscosity in the model.

In Figure 5(a), the calculation results for the hole migration through the sequence A1 T2 C3 G4 A5 (+) C6 A7 A8 A9 in liquid water $\left(\alpha_{n}^{\prime}=0.249 \mathrm{eV} / \AA\right)$ are shown. Since the case is considered when the selected DNA segment is crossed by the particle's track, there is a high probability of double-strand break induction when both strands of the DNA molecule can be broken in this area (Figure 4). However, it is assumed that the $\pi$-orbitals between DNA bases overlap at the initial time in a manner enabling the charge migration along the chain.

The process is initially unstable and reversible, as indicated by the oscillations of $P_{5}(t), P_{7}(t)$ and $P_{8}(t)$. At $t \sim 1.7 \cdot 10^{-11} \mathrm{~s}$ the probabilities stabilize. The probability of the charge to be located at the initial base A5(+) decreases considerably. Then the hole localizes preferentially on the bases A7 and A8. The probability of the hole to be located on the bases A1, T2, C3, G4, C6, and A9 is rather low during the period under consideration. Since the bases A5, A7, A8 are separated by the base C6, these results suggest the possibility of a quantum tunnel effect. Indeed, this effect was observed experimentally for hole migration in short DNA sequences like $\mathrm{G}(+)$ T G G G, G(+) A T G G G, or G(+) A T T G G G [1, 2]. In these cases, despite the fact that the ionization potential of guanine is less than that of thymine $T$ and adenine $\mathrm{A}$, the hole migrates from the guanine $\mathrm{G}(+)$ to the guanines G G G bypassing adenine and thymine. In these sequences, cytosine $\mathrm{C} 6$ represents a potential barrier for the charge (i.e. the ionization energies of adenines A5, A7, A8 are less than that of cytosine C6).

Similarly, calculations for the sequence A1 T2 C3 G4 A5(+) C6 A7 A8 A9 in the gas phase $\left(\alpha_{n}^{\prime}=\right.$ $0.119 \mathrm{eV} / \AA$ ) is performed (Figure 5(b)). In this case, the charge is localized on the four adenines A5(+), A7, A8, and A9 shortly after irradiation. Then the hole migrates to the first adenine A1 and locates there. 

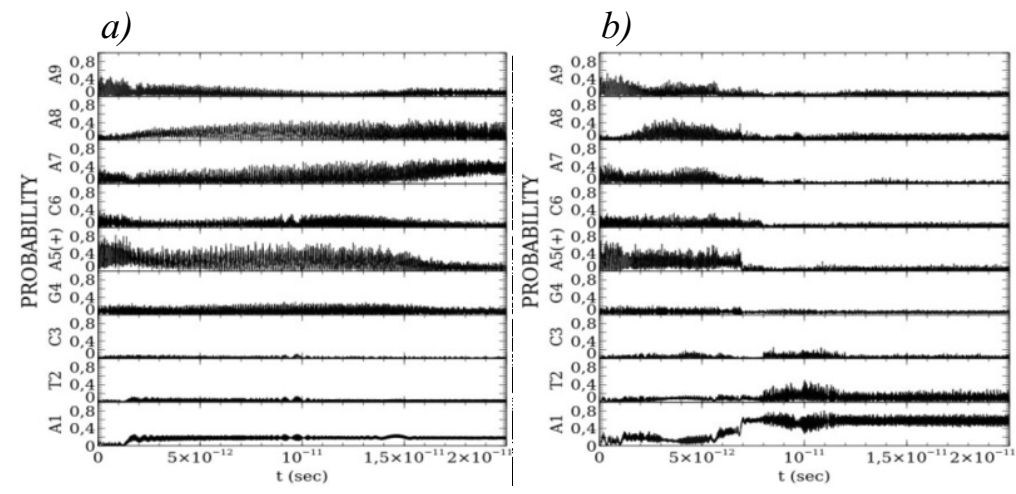

Fig. 5. Time evolution of the probabilities of hole location $P_{n}(t)$ at the bases for A1 T2 C3 G4 A5(+) C6 A7 A8 A9 sequence: (a) liquid phase; (b) gas phase.

\section{Conclusion}

The results of the work indicate that the possible sites of oxidative damage in DNA may be dependent on the environment conditions and are different for the aqueous and gas medium. The environmental properties of the medium are introduced in the model by using known values of the viscosity, which influence the damping parameter. Indeed, the values of $P n(t)$ are very sensitive to the latter. In liquid water, the time-dependent redistribution of a charge occurs mainly between the bases A7 and A8 at the final simulation time (Figure 5(a)). In the gas phase, the charge is distributed mainly between A5, A7, A8, and A9 at first, and then it migrates to the base A1 and remains at this location (Figure 5(b)).

The model also puts in evidence the importance of the quantum nature of charge migration in DNA. Therefore, DNA damage process should not be considered only from classical models. A quantum tunnel effect is suggested for the cases shown in Figure 5 when a hole hops from A5(+) to adenines A7, A8 (Figure5(a)) and A1 (Figure 5(b)), effectively crossing their respective potential barriers caused by cytosine $\mathrm{C} 6$, as well as cytosine $\mathrm{C} 3$ and thymine T2, respectively. Here, the charges evolve as demonstrated in experiments $[1,2]$ when they migrate through bases possessing bases with higher ionization energies than initial and final bases.

It is assumed that the investigation demonstrates the utilization of formalism for the study of the charge migration through different molecular sequences that may be tested in experiments.

\section{Acknowledgments}

This research was supported by the SC IACP DVO RAS and the JINR Grant \# 14091-01. This project is also supported by the Far Eastern Federal University, Grant 13-09-617-m_a and by the NASA Space Radiation Program (NAS9-02078).

\section{References}

[1] E. Meggers, M. Michel-Beyerle and B. Giese, Sequence dependent long range hole transport in DNA, Journal of the American Chemical Society 120 (1998), 12950-12955. 
[2] B. Giese B, S. Wessely, M. Spormann, U. Lindemann, E. Meggers and M.E. Michel-Beyerle, On the mechanism of long-range electron transfer through DNA, Angewandte Chemie International Edition 38 (1999), 996-998.

[3] V.D. Lakhno, Soliton-like solutions and electron transfer in DNA, Journal of Biological Physics 26 (2000), 133-147.

[4] S. Eh. Shirmovsky and D.L. Boyda, Study of DNA conducting properties: Reversible and irreversible evolution, Biophysical Chemistry 180-181 (2013), 95-101.

[5] C.T. Shih, Sequence and energy dependence of electric transport properties of DNA -A tight-binding model study, Chinese Journal of Physics 45 (2007), 703-707.

[6] P. O'Neill, A.W. Parker, M.A. Plumb and L.D.A. Siebbeles, Guanine modifications following ionization of DNA occurs predominantly via intra- and not inter-strand charge migration: An experimental and theoretical study, Journal of Physical Chemistry B 105 (2001), 5283-5290.

[7] K. Senthilkumar, F.C. Grozema, C. Fonseca Guerra, F.M. Bickelhaupt and L.D.A. Siebbeles, Mapping the sites for selective oxidation of guanines in DNA, Journal of the American Chemical Society 125 (2003), 13658-13659.

[8] M. Dizdaroglu, Mechanisms of oxidative DNA damage; lesions and their measurement, In: Advances in DNA Damage and Repair, M. Dizdaroglu and A.E. Karakaya, Eds., Kluwer Academic, Plenum Publishers, New York, 1999. pp. 67-87.

[9] M. Hada and A.G. Georgakilas, Formation of clustered DNA damage after high-LET irradiation: A review, Journal of Radiation Research 49 (2008), 203-210.

[10] R. Morita, S. Nakane, A. Shimada, M. Inoue, H. Iino, T. Wakamatsu, K. Fukui, N. Nakagawa, R. Masui and S. Kuramitsu, Molecular mechanisms of the whole DNA repair system: A comparison of bacterial and eukaryotic systems, Journal of Nucleic Acids 2010 (2010), 179-594.

[11] Wenwei Tanga, Min Zhang and Xinping Zeng, Establishment of dsDNA/GNs/chit/GCE biosensor and electrochemical study on interaction between 6-mercaptopurine and DNA, Bio-Medical Materials and Engineering 24 (2014), 10711077.

[12] A. Adhikary, A. Kumar and M.D. Sevilla, Photo-induced hole transfer from base to sugar in DNA: Relationship to primary radiation damage, Radiation Research 165 (2006), 479-484.

[13] W. Zhang, R. Yang and S.E. Ulloa, Charge transport in DNA molecules: Cooperative interplay between the disordered base-pair channel and the ordered backbone, Physical Review E 80 (2009), 051901.

[14] D.D. Eley and D. Spivey, Semiconductivity of organic substances, Part 9 nucleic acid in the dry state, Transactions of the Faraday Society 58 (1962), 411-415.

[15] I. Plante and F.A. Cucinotta, Ionization and excitation cross sections for the interaction of HZE particles in liquid water and application to Monte Carlo simulation of radiation tracks, New Journal of Physics 10 (2008), 1-15.

[16] I. Plante and F.A. Cucinotta, Cross sections for the interactions of $1 \mathrm{eV}-100 \mathrm{MeV}$ electrons in liquid water and application to Monte-Carlo simulation of HZE radiation tracks, New Journal of Physics 11 (2009), 1-24.

[17] I. Plante and F.A. Cucinotta, Monte-Carlo simulation of ionizing radiation tracks, in: Applications of Monte Carlo Methods in Biology, Medicine and Other Fields of Science, C.B. Mode ed., InTech, Rijeka, Croatia, 2011, pp. 315-356.

[18] X.-J. Lu and W.K. Olson, 3DNA: A software package for the analysis, rebuilding and visualization of threedimensional nucleic acid structures, Nucleic Acids Research 31 (2003), 5108-5121.

[19] F. Lewis and Y. Wu, Dynamics of superexchange photoinduced electron transfer in duplex DNA, Journal of Photochemistry and Photobiology C 2 (2011), 1-16.

[20] T. Kubar, P.B. Woiczikowski, G. Cuniberti and M. Elstner, Efficient calculation of charge-transfer matrix elements for hole transfer in DNA, Journal of Physical Chemistry B 112 (2008), 7937-7947.

[21] V.D. Lakhno, Dynamics of the hole transfer in the nucleotide sequences, in: Computers and Supercomputers in Biology, V.D. Lakhno and M.N. Ustinin, eds., Institute of Computer Investigations, Moscow-Izhevsk, 2002, pp. 137-167.

[22] T. Holstein, Studies of polaron motion: Part II. the "small" polaron, Annals of Physics 8 (1959), 343-389.

[23] A.A. Voityuk, Electronic couplings and on-site energies for hole transfer in DNA: Systematic quantum mechani$\mathrm{cal} /$ molecular dynamic study, Journal of Chemical Physics 128 (2008), 115101-115106.

[24] L.V. Yakushevich, Nonlinear Physics of DNA, Wiley-Verlaq GmbH \& Co. KGaA, Weinheim, 2004. 